\title{
ANÁLISIS DEL ABORTO DESDE UN PUNTO DE VISTA CONCEPTUAL

\section{Resumen}

El aborto se sitúa como uno de los más graves atentados contra la solidaridad intergeneracional porque ataca directamente la filiación. La diacronía establece el amparo del más débil y el amor entre padres e hijos. Esto se rompe desde las amenazas a la figura del hijo. Este artículo se desarrolla en el plano de los conceptos y la necesidad de contribuir a la vida en la sociedad actual. Para ello es necesario incidir en la dignidad de la vida humana en aquellos círculos académicos y de debate intelectual desde donde se crean las distintas corrientes de opinión.

Palabras clave (fuente: DeCS): filiación; aborto; familia; sociedad; control social formal.

\section{DOI: 10.5294/PEBI.2019.23.1.3}

\section{PARA CITAR ESTE ARTíCULO / TO REFERENCE THIS ARTICLE / PARA CITAR ESTE ARTIGO}

Aznar-Sala FJ. Análisis del aborto desde un punto de vista conceptual. Pers Bioet. 2019; 23(1): 34-48. DOI: https://doi.org/10.5294/pebi.2019.23.1.3

1 orcid.org/0000-0003-0510-0425. Universidad Católica de Valencia “San Vicente Mártir”, España. fjavier.aznar@ucv.es

RECEPCIÓN: 19/07/2018

ENVÍO A PARES: 10/09/2018

APROBACIÓN POR PARES: 08/10/2018

ACEPTACIÓN: 01/11/2018 


\section{Abstract}

Abortion is one of the most serious attacks against intergenerational solidarity because it directly attacks filiation. The diachrony establishes the protection of the weakest and the love between parents and children. This is broken from the attacks on the figure of the child. This paper moves in the concepts and the need to contribute in favor of life in today's society. For this, it is necessary to influence in favor of the dignity of human life in the academic and debate circles, spaces in which the different currents of opinion are created.

Keywords (source: DeCS): Filiation; abortion; family; society; social control, formal.

\section{Resumo}

O aborto posiciona-se como um dos mais graves atentados contra a solidariedade intergeracional, porque ataca diretamente a filiação. A diacronia estabelece o amparo do mais fraco e o amor entre pais e filhos. Isso se quebra a partir das ameaças à figura do filho. Este artigo se desenvolve no plano dos conceitos e da necessidade de contribuir para a vida na sociedade atual. Para isso, é necessário incidir na dignidade da vida humana nos círculos acadêmicos e de debate intelectual, contextos em que são criadas as diferentes correntes de opinião.

Palavras-chave (fonte: DeCS): Filiação; aborto; família; sociedade; controle social formal. 


\section{INTRODUCCIÓN}

El propósito de este artículo es situar la investigación sobre la realidad del aborto en el mundo desde la perspectiva de las ciencias sociales y en diálogo con la obra del sociólogo español José Pérez Adán. La propuesta, aunque parte de una definición biológica, se dirige in extenso al debate conceptual propio de las ciencias sociales e intenta aportar luz sobre las consecuencias que en el plano social e intelectual supone la aceptación del aborto. La obra de Pérez Adán sobre este supuesto, y su lucha por la defensa de la vida durante toda su trayectoria académica, hacen de este autor un referente necesario dentro de la protección de la vida humana. De este modo, de todo su bagaje académico se deduce que la aceptación social del aborto se vuelve contra el propio hombre y supone una de las mayores transgresiones contra la familia y la sociedad. En el presente escrito iremos desglosando progresivamente cuáles son las conciencias que se derivan de este hecho y de su aceptación en el plano social. No en vano, la admisión del aborto por parte de una sociedad poco informada al respecto es una de las problemáticas más difíciles de superar entre aquellos académicos que buscan argumentar científicamente la inconveniencia de este hecho en todos los niveles humanos.

\section{LA SOCIEDAD COMO DIACRONÍA}

Es necesario, en un primer instante, definir qué es el aborto desde el punto de vista bilógico, para después abordar el problema que deriva de su aceptación desde el punto de vista de las ciencias sociales. Para el doctor Justo Aznar, el aborto deliberadamente provocado consiste en la eliminación voluntaria de una vida que ya es y que:
... no es la simple suma de dos células germinales. Se ha constituido una nueva realidad biológica, que empieza a operar de forma unitaria, que en vez de dirigirse a la muerte tiene vida propia, es un nuevo ser bilógico, y en este caso, por proceder de gametos humanos, un nuevo ser humano. Estamos ante el embrión de una sola célula, que después se desarrollará a embrión preimplantado [si no se le niega el derecho a nacer mediante la práctica del aborto] (1).

Desde el plano social, una vez se ha especificado el dato biológico, el aborto conlleva dejar a la familia mutilada, pues algo relativo a la vida biológica es la relación familiar en el sentido de ser: hijo/a, padre/madre, amigo/a, esposo/a, abuelo/a. Sin este nudo biográfico-y, a la vez, diacrónico- de sucesivas relaciones humanas, estudiaríamos al hombre sin comprender en plenitud que "los seres humanos no somos sociales después de nacer a la vida, en todo caso lo somos antes: basta pensar que nacemos en el marco de una relación de filiación" (2). Como señala el filósofo español Julián Marías (19142005), para reforzar esta primera aseveración: “La razón es que mi vida acontece en forma de convivencia [...] en mi vida encuentro otras vidas. Me descubro como un yo frente a un tú" (3). Así pues, un hecho definitorio del ser humano es el de pertenecer a una familia y a un entramado de relaciones afectivas, tanto horizontal como verticalmente. Las relaciones horizontales se establecen en relación con nuestros antepasados y, con el tiempo, se tornan verticales cuando por el paso de los años se pasa al estado de padre o de abuelo. Las relaciones horizontales se conforman con aquellos grupos humanos que elegimos en el sentido de la amistad, el ocio, el trabajo o el afecto depositado en una persona concreta, o como proyecto de amor y familia, mientras que las verticales 


\section{LA SALUD DE UNA SOCIEDAD NO ESTÁ DEFINIDA POR MÁS O MENOS ESTADO, O MÁS O MENOS REGULACIÓN DE MERCADO: LA SALUD SOCIAL SE DEFINE POR LA MAYOR O MENOR ADECUACIÓN \\ DE UNA SOCIEDAD A CRITERIOS MORALES DE EXCELENCIA.}

nos vienen dadas por pertenecer como seres humanos a un núcleo familiar. De esta manera, la familia entraña en sí misma el lugar de crecimiento y de seguridad que un ser humano precisa para desarrollarse como tal, ya que sin ella sería imposible establecer un marco de solidaridad indispensable para el hombre. Desde el punto de vista biológico, el hombre es un ser deficiente y menesteroso que, nacido prematuramente, necesita madurar y construir a su alrededor un mundo de cultura (4). No faltan quienes, desde una perspectiva individualista, vienen a difuminar los límites del grupo familiar/ social y abogan por otros posibles tipos de agrupación humana o multifamilismo (5), pero no nos detendremos ahora es este tipo de agrupaciones alternativas.

La familia posee, como carácter primigenio, el débito del amor y de la ayuda intergeneracional dentro de un marco de diacronía que se establece recíprocamente entre padres e hijos. Se nace en familias que ya existen, y la filiación se presenta como algo radical y universal en la vida de todo ser humano, desde donde se es reconocido y donde nadie se siente extraño, de no ser alguien ajeno a este sustantivo núcleo familiar (6). La progresiva disfunción familiar, hacia la que la sociedad deriva, conlleva determinadas consecuencias que serán objeto de análisis en el presente estudio, tales como el descenso alarmante de la natalidad -que viene ayudado por la dramática realidad del aborto-, la soledad de muchos hombres y mujeres, y el posible abandono de los mayores, así como el alto índice de rupturas familiares.
Todo lo referido se presenta, en su conjunto, como uno de los síntomas más alarmantes de la progresiva disfuncionalidad familiar que aboca a que el egoísmo prevalezca frente a la caridad y a la creciente pérdida de valores sociales. Los males de nuestra sociedad actual quedan referidos a sus miembros, pues como se subraya desde la sociología comunitarista - defendida por el economista Amitai Etzioni y seguida por Pérez Adán-, no basta con medir el progreso social en términos de bienestar o mercantiles, el criterio máximo ha de establecerse desde la salud social que está en conexión con la virtud como principal elemento de valoración donde prime el valor humano y no únicamente el de renta per cápita: "La salud de una sociedad no está definida por más o menos Estado, o más o menos regulación de mercado: la salud social se define por la mayor o menor adecuación de una sociedad a criterios morales de excelencia" (2).

La paternidad es sustantiva en referencia al hijo y desde la perspectiva sociológica, pues no tiene sentido en sí misma sino en correspondencia con la vida que proviene del amor de unos padres, se trata de un amor en relación con el hijo que está en la base de la virtud social: "El niño no nacido aún es una realidad 'viniente', que llegará si no lo paramos" (7). Si la filiación es atacada en su matriz y se instalan todo tipo de antiideologías en el nivel conceptual, se excluye del mismo modo la paternidad porque lo más básico y obvio en el hombre y en la mujer es la filiación, pues es seguro que todo ser humano es hijo y no existe la paternidad sin esta 
asociación, pero no es tan seguro que todos sean padres (biológicamente hablando). No todos serán necesariamente padres en su trayectoria vital pero, en cambio, todos son hijos, y se trata de un hecho referencial que es común a todo el género humano sin excepción: “[el hijo] es un 'tercero' absolutamente nuevo, que se añade al padre y a la madre" (7). Repensar la familia, desde la diacronía, pasa a ser una de nuestras primeras obligaciones como investigadores de las ciencias sociales y sitúa la mirada en la necesidad de incidir positivamente en la sociedad a corto, medio y largo plazo: "La sociedad no puede perseverar en mirar para otro lado como si nada estuviese ocurriendo, como si la principal causa de muerte en varias naciones no existiese. Es algo con lo que nos tenemos que enfrentar y ante lo que hay que posicionarse del lado de las víctimas" (8).

\section{LA IMPORTANCIA DEL “HIJO" EN LA ESTRUCTURA FAMILIAR}

Como hemos advertido, desde una perspectiva social, lo común al ser humano, además de la naturaleza, es la realidad incuestionable de la filiación, es decir, todos somos hijos de nuestros respectivos padres. Esa filiación conforma la diacronía y las referencias familiares. La diacronía, como se indicaba desde el terreno de las ciencias sociales, "supone un diálogo intergeneracional que asume el futuro de nuestros hijos y sus aspiraciones" (5). Julián Marías, en la Tribuna Abierta de $A B C$, al reflexionar sobre el concepto de diacronía y filiación, diría lo siguiente: "El nacimiento de un niño es una radical 'innovación de la realidad': la aparición de una realidad 'nueva'. Se dirá que se deriva o viene de sus padres. Sí, de sus padres, de sus abuelos y de todos sus antepasados" (7). No olvidemos, como contrastan los estudios biológicos, que "el embrión es realidad humana, individuo de la especie, persona, desde que es cigoto, porque posee toda la información del sistema respecto al término: tiene como propia la capacidad de un desarrollo orgánico. Y actualizará en cada tiempo de su vida toda la información de ese momento vital" (9). El dato que nos proporciona la ciencia mediante la biología refuerza nuestro argumento sociológico que también posee su propio método científico y que converge con el primero en el sentido de la humanidad y lo que significa el derecho de todo ser humano a la vida, pues no son pocos los ambientes donde primero se despersonaliza la vida humana incipiente para después negarla: "La cultura actual tiene varias aristas, algunas de las cuales tienden a lesionar al ser humano, o al menos a tratarlo como a cualquier otra criatura, separándolo de la dignidad que le es propia y de la que no se le debe despojar" (10).

El modelo que nos propone la sociología comunitarista, al que ya hemos aludido líneas arriba, implica la realidad familiar en su conjunto y permite que hablemos de sujeto colectivo en vez de sujeto individual. El sujeto colectivo posee mayor riqueza en el plano social que el individual, en tanto que supone la apertura hacia otro que no soy yo y que me complementa, mientras que el modelo individualista invita a replegarse sobre el propio sujeto sin la referencia necesaria a la apertura. Un dato constitutivo de lo humano, y que nos enriquece, es nuestra herencia familiar, que a su vez reproducimos como un bien propio y de aquellos que con el tiempo quedan socialmente a nuestro cargo. Más allá de la herencia biológica encontramos la responsabilidad del débito del amor y de la ayuda entre generaciones. En la familia nacemos a un horizonte plagado de relaciones que encuentran su equilibrio desde el origen en esta agrupación. Por tanto, algo completamente extraño a la institución familiar sería el uso de la violencia, cuando de tal institución se 


\section{LA INCIDENCIA EN EL TERRENO CONCEPTUAL} ES MÁS IMPORTANTE DE LO QUE PODRÍAMOS PENSAR, PUES LA ACEPTACIÓN DE UN TIPO DE VISIÓN REDUCCIONISTA DE LA REALIDAD ES LO QUE A LA POSTRE LE DA LEGITIMIDAD A UNA DETERMINADA CORRIENTE DE OPINIÓN.

espera la protección y el amparo, de ahí que el presente artículo intente aportar luz sobre las disfunciones sociales que pueden presentarse en la familia y el porqué de las mismas. Siguiendo este postulado, hay que reseñar que la familia posee en esencia cuatro notas características -a juicio del sociólogo Pérez Adán-: a) equidad generacional, b) socialización, c) transmisión cultural y d) control social (6). Pues bien, la realidad del aborto rompe todas y cada una de estas cuatro notas. En un primer momento quiebra la equidad generacional porque se malogra el cuidado entre las distintas generaciones, que no solo afecta al feto, sino que tiene repercusión en la falta de cuidados en la ancianidad o en la enfermedad, pues la sociedad se deshumaniza desde la matriz y después apunta a la eutanasia como solución definitiva. Afecta también a la socialización, pues de forma evidente se deja de socializar a un ser humano que lo merece en justicia, e incluso se le niega la propia vida en el seno familiar. Es más, la comunicación humana que supone la inserción cultural queda quebrada y no es posible ejercer el control social porque en la raíz societaria se inserta la violencia hacia el más débil que necesita protección y cuidado. Por ello, el aborto y la eutanasia siguen una misma lógica, primero la muerte del hijo y después la muerte del padre, pues el padre no tiene sentido sin la figura del hijo, una influye de forma decisiva a la otra.
No solo deja de darse la posibilidad de educar al hijo de futuras desviaciones, sino que se inserta socialmente y se legitima mediante el aborto la justificación de la desviación de futuras generaciones que perciben nítidamente cómo se cosifica conceptualmente al ser humano. Es curioso que en la actualidad se hable tanto de violencia entre la juventud y nadie sepa cómo ponerle freno a estas conductas desviadas que irrumpen en la familia y en las aulas. No en vano se acrecientan los síntomas propios de enfermedades del alma, como el caso de la soledad, que incide directamente en la causa de las patologías en la ancianidad; en recientes estudios de la Universidad de Helsinki se dice que: "El aislamiento social, de forma similar a otros factores de riesgo como la depresión, puede considerarse como un factor de riesgo para el mal pronóstico de las personas con enfermedad" (11). De este modo, la salud social se debilita y se hace necesario reconocer, a la luz de los síntomas sociales, que "una proliferación de muertes prematuras puede ocasionar graves disfunciones sociales" (12).

\section{EL "ABORTO CONCEPTUAL" VA CONTRA “LA VIDA DEL HIJO" A NIVEL CONCEPTUAL}

La incidencia en el terreno conceptual es más importante de lo que podríamos pensar, pues la aceptación de un tipo de visión reduccionista de la realidad es lo que a la postre le da legitimidad a una determinada corriente de opinión. De hecho, aquellos ideólogos que se presentan como defensores del aborto saben que esto es así y por ello se niegan a entrar en determinadas terminologías en las que podrían sentirse incómodos. Por ejemplo, para abordar esta cuestión se sirven de eufemismos que significan etimológicamente cosas distintas a la realidad señalada (en griego eu significa bien y feme significa hablar, precisamente hablar bien). Los eufemismos 
buscan ser dulces al oído, aunque lo que escondan sea horrible en la praxis, por ello es una primera derrota admitir este primer juego lingüístico: "Una vez oí hablar a Bernard Nathanson y decir que la ingeniera de las palabras es el primer paso de la ingeniería social" (8). Desde este punto de vista se puede hablar bien pero obrar mal, pues la recta habla no va ligada necesariamente a la recta conciencia: "Entre los conceptos cuya terminología debe rechazarse por razones de coyuntura y conveniencia política pensamos que están: a) conceptos engañosos como los supuestamente asépticos de interrupción del embarazado', o de 'reducción embrionaria'; y b) conceptos contradictorios como el de 'salud reproductiva' que incluye el aborto en la terminología de los organismos internacionales, o el de ‘aborto terapéutico"” (8). De este modo, "una persona recta y un científico riguroso jamás pueden usar una terminología que esconda violencia y víctimas, y deberá incluir en su diccionario de buenas prácticas el uso de vocablos alternativos a los considerados por algunos como "políticamente correctos"” (8).

Lo que se opone a esta realidad social de la filiación, inserta en lo más profundo de la dimensión humana y que supone una ruptura "conceptual" de esa cadena ininterrumpida de filiaciones, es justamente la dura realidad del aborto. Este hecho cosifica a un ser humano al que se puede utilizar en adelante, e impide conceptualmente que nos reconozcamos como lo que somos: personas. Dirá Julián Marías al hilo de la posible cosificación de la persona lo siguiente: "Esta visión ha de fundarse en la distinción entre 'cosa' y 'persona', tal como aparece en el uso de la lengua. Todo el mundo distingue, sin la menor posibilidad de confusión, entre ‘qué’ y ‘quién', 'algo' y ‘alguien', 'nada' y 'nadie”' (7). Con ello se trunca en el plano teórico una nota esencial y constitutiva de lo humano que nos permite reconocernos. El aborto se sitúa de forma mayestática en la negación del hijo, pero cualquier otra práctica que lo niegue, como los úteros artificiales, donde las mujeres no necesiten dar a luz para ser madres, o las fecundaciones in vitro vendrán a dificultar desde el origen la relación paterno-filial, lo que rebaja lo humano y nos transporta hacia el ser-producidos y no seres-procreados donde el hijo no es engendrado como un acto de amor sino producido como un deseo personal:

¿No estará en curso un proceso de “despersonalización”, es decir, de "deshominización" del hombre y de la mujer, las dos formas irreductibles, mutuamente necesarias, en que se realiza la vida humana? Si las relaciones de maternidad y paternidad quedan abolidas, si la relación entre los padres queda reducida a una mera función biológica sin perduración más allá del acto de generación, sin ninguna significación personal entre las tres personas implicadas, ¿qué queda de humano en todo ello? (7).

La explicación conceptual de lo humano se asienta indisociablemente en la filiación, por lo que el problema del aborto es un problema de justificación racional. El aborto es un problema de difícil justificación racional y humana y se sitúa como una de las más terribles antiideologías en tanto que es algo ilógico y contra-razón. Si 
situamos el aborto en el plano del derecho destruimos las bases y los fundamentos de nuestra vida como sociedad. El ser del hijo, junto con la filiación en referencia a la familia, es lo que al ser humano le hace tal y esto empieza a ser realmente cuestionable con la aceptación social del aborto que niega todo lo anterior: "El núcleo de la cuestión es la negación del carácter personal del hombre. Por eso se olvida la paternidad y se reduce la maternidad a soportar un crecimiento intruso, que se puede eliminar. Se descarta todo uso del 'quién', de los pronombres tú y yo" (7).

Tal realidad viene a romper la generosidad intergeneracional porque esta supone que las clases activas velan por las clases pasivas, especialmente cuando estas no pueden valerse por sí mismas. Si la clase pasiva -el niño engendrado no nacido- es eliminada, este amparo y solidaridad intergeneracional se eliminan de raíz. Cabe resaltar que hablamos desde los planos teórico y conceptual con el ánimo de contrarrestar aquellas opiniones contrarias. No entramos en este escrito en determinadas casuísticas que puedan presentarse desde el campo de la medicina o del derecho, y que merecerán una valoración adecuada al caso concreto. No obstante, es importante resaltar que una sociedad no puede vivir humanamente si acepta el derecho al aborto como idea marco, como tampoco podemos convivir como sociedad con antiideologías cercanas a la defensa de la supremacía racial o que omitan la defensa de ciertas minorías, pues, de algún modo, seríamos participes de tal disfunción social. Todo ello supone una ruptura de la solidaridad que se establece a través del tiempo y significa, a su vez, apropiarse de un derecho que no nos pertenece y sí pertenece a otros (8). Desgraciadamente, nunca como antes las decisiones de la política sobre la vida humana han sido de tan enorme calado. Las distintas sociedades han tendido a la uniformidad y se han vuelto excesivamente dependientes de los centros de poder político. En España, por ejemplo, se practican más de 100.000 abortos al año, y eso que la pirámide poblacional en cuanto a la natalidad está entre las tres más bajas del mundo, con $1,33 \%$ de nacimientos por año, cuando sabemos que para que se dé el adecuado relevo generacional hace falta una tasa de 2,11\% por familia (13). En la actualidad este hecho ha provocado, desde posturas económicas, un acalorado debate sobre la dificultad para sufragar las futuras pensiones de los mayores y la imposibilidad de sostener el actual sistema de financiación de las mismas y, con ello, el Estado de bienestar. Desgraciadamente, nadie afronta la realidad hablando del aborto y de la necesidad de revertir las disfunciones sociales actuales que conducen hacia un progresivo envejecimiento de la población española y un déficit de solidaridad que se ha instalado conceptualmente y parece ser aceptado sin la crítica necesaria. Esto ya se ha advertido desde la sociología, y es necesario que se sumen más voces desde distintos ámbitos científicos, donde se señale la raíz de estos males, pues "muy pocos países podrán hacer frente en los próximos años al cuidado de una población cada vez más envejecida” (10).

\section{EL ABORTISMO ES UNA IDEOLOGÍA ANTISOCIAL QUE ROMPE LA SOLIDARIDAD INTERGENERACIONAL}

El actual imaginario social en el que nos movemos, y desde cuya perspectiva el aborto es visto como algo neutro, nos deshumaniza como sociedad y, por ello, se hace necesario generar sinergias de opinión favorables de la dignidad de toda vida humana desde su inicio natural al fin último de la misma. Se trata de un tema que, sin criminalizar a ningún sector social que opine 
distinto, ayudaría a una discusión de mayor calado. En los planos intelectual y académico es una cuestión que necesita de un amplio, sosegado y generoso debate por la importancia del mismo. La realidad actual nos aboca a una serie de preguntas que es obligado responder, la primera de ellas sería: ¿cuál es el futuro que visionamos desde nuestro análisis sociológico? En primer lugar, afirmar nuestro deseo de que, como sociedad que busca caminar hacia la excelencia, puedan surgir países que consideren ilegítimo el aborto, o sea, que haya cada vez más sociedades que se sumen a la defensa de la vida y denuncien como vergonzosa esta práctica. En un segundo momento, deseamos que esos mismos países influyan en aquellos Estados que sí permiten el aborto para que la vida sea salvaguardada como un derecho humano. Esta es, y no otra, la gran conquista que nos queda y de la que todos somos deudores en nuestros ámbitos de influencia. La gran desigualdad actual en el mundo no es la de aquellos que más tienen frente a los que menos tienen, o sea, la diferencia clásica entre Norte y Sur, sino la dramática diferencia de los que pueden nacer y los que no pueden nacer porque no se les permite este derecho inalienable, pues ya son vidas humanas. Esta es la gran conquista en el presente. En un mundo que pretende acabar con las grandes desigualdades y parece cada vez más concienciado y sensibilizado ante ellas, esta es la primera gran desigualdad por superar y sobre la que hay que debatir con mayor vigor. Desde esta perspectiva hay que poner el foco sobre aquellos líderes mundiales y leyes permisivas que justifican el aborto para identificarlas como mala praxis médica y sociológica:

Algunas personas justifican el aborto y otras prácticas inhumanas de control de la natalidad con base en la carencia de recursos que tienen los más pobres. Algunos como el prestigioso economista H. Daly y otros expertos del Banco Mundial, han llegado incluso a hablar de la necesidad de establecer un consenso sobre el número de generaciones que van a poblar la tierra ante el inevitable colapso que va a producir la falta de balance energético (12).

En países como China, uno de los ejemplos que más sobresalen, se dispara el número de abortos practicados en la actualidad y todo ello amparado por el Estado: "En China se realizan unos 13 millones de abortos al año, según el Centro de Investigación de Tecnología bajo la Comisión Nacional de Planificación Familiar y de la Salud" (14). Este dato conlleva una grave inestabilidad poblacional: "con los años se da un envejecimiento de la población y uno de los peores desequilibrios en el número de hombres y mujeres del mundo, debido sobre todo a los abortos selectivos de fetos femeninos" (15). Desafortunadamente, los países con más peso en el mundo, y que decretan embargos y expulsan a embajadores con malas praxis democráticas o por no defender la libertad y los derechos humanos en pleno siglo XXI, se caracterizan por el silencio ante el terrible drama de las cifras abortivas que se suceden año tras año en países como los citados, y a los que no se atreven a denunciar por miedo a perder cuotas de mercado:

Según se publica en The Lancet el número de abortos aumenta en el mundo, habiendo llegado en 2015 a 56 millones, cifra ciertamente escandalizadora si se considera que con cada aborto se pierde una vida humana. Habría que conocer si existe alguna otra causa, no relacionada con enfermedades humanas, que cause mayor mortalidad que el aborto (16). 


\section{UNA ANTIIDEOLOGÍA ES, POR NECESIDAD, UN TIPO DE PENSAMIENTO QUE VA CONTRA LA DIGNIDAD DE LA VIDA HUMANA Y NO SE AJUSTA A LA RAZÓN LÓGICA DEL BIEN, POR ELLO DEBE SER SUPERADA EN UNA SOCIEDAD QUE BUSQUE LA PERFECCIÓN Y NO ÚNICAMENTE EL PROGRESO EN RENTA PER CAPITA.}

También se refiere en el artículo que el número de abortos entre 1990 y 1994 era aproximadamente de 50 millones, cifra que ha pasado a 56 entre 2010 a 2014 :

Este aumento se debe especialmente a su aumento en los países en vías de desarrollo, pues en los países ricos ha disminuido desde 25 abortos por 1000 mujeres en edad reproductiva a 14 por las mismas 1000 mujeres. También cabe destacar que el área geográfica en la que se dan más abortos es Latinoamérica donde aproximadamente se produce un aborto por cada tres embarazos (16).

Tal hecho conlleva una concatenación de causas de inexorable disfunción familiar que desembocarían en planteamientos eutanásicos que ya surgen en muchos países. De hecho, los mayores abandonados por sus hijos son cada vez más y representan un serio desafío a las modernas sociedades que no saben cómo dar solución a un problema que aumenta día tras día, pues desde la matriz de nuestra sociedad se gesta el egoísmo y no la generosidad:

Algunos alegan que la casa en la que viven es pequeña. Otros, que tienen mucho trabajo y nada de tiempo. Y algunos ni siquiera se molestan en poner una excusa: directamente no responden a las llamadas. La sala de Urgencias del Hospital General se ha convertido en un lugar utilizado por familiares que abandonan a sus mayores cuando son un estorbo en casa (17).

\section{NECESIDAD DE PREVENIRNOS CONTRA TODO TIPO DE ANTIIDEOLOGÍAS}

Una antiideología es, por necesidad, un tipo de pensamiento que va contra la dignidad de la vida humana y no se ajusta a la razón lógica del bien, por ello debe ser superada en una sociedad que busque la perfección y no únicamente el progreso en renta per capita. En este sentido, el pensador español Manuel García Morente (1886-1942), alertando sobre uno de los mayores peligros de nuestra sociedad, dirá que "el progreso no consiste en el ser más sino en el valer más, en el ser mejor” (18). Además, uno de los más brillantes sociólogos franceses e impulsor de la sociología pragmática, Luc Boltanski, se ha planteado el tema del aborto sin recurrir a ideologías ni consignas, y considera que desgraciadamente las modernas sociedades no buscan la perfección en los valores sino únicamente el progreso económico. Boltanski no defiende la vida, pero se plantea el problema y hace que se lo planteen a su vez la Gauche divine y el establishment o casta académica francesa (19). No olvidemos que "el vínculo intergeneracional es socialmente constituyente y, si se ignora, como hace normalmente el individualismo, se ignora a la sociedad en sí misma. Es decir, se ignora también lo que 'no existe' (las futuras generaciones, por ejemplo)” (10). El catedrático de filosofía del derecho, 
Jesús Ballesteros, incluye el aborto como uno de los problemas donde se ejerce también la violencia doméstica y subraya lo siguiente: "erradicar completamente la violencia doméstica supone eliminar el pretendido derecho de propiedad de unos seres humanos sobre otros, sea el varón sobre la mujer, sean los padres sobre los hijos" (20). El aborto priva a la sociedad de la riqueza del otro y rompe la dependencia entre sujeto y colectividad, pues lo realmente radical en el sujeto humano no es solo que sea, sino que coexista. La gestación de una criatura es un hecho social relevante y situarse frente a ello por medio de la eliminación del hijo obliga a dejar en suspenso la razón y no afrontar decidida y racionalmente uno de los mayores problemas que como colectivo humano se nos presenta. En la actualidad se da una corriente de opinión favorable al aborto, y todos así lo percibimos, muchas veces con notables dificultades en su defensa por la presión social establecida y el poder de los medios de comunicación; pero si encaramos desde la lógica y la razón esta cuestión, es muy posible que pueda revertirse paulatinamente dicha corriente de opinión porque la verdad acabará imponiéndose por su belleza. Sabemos que los distintos Estados legislan a golpe de encuestas de opinión y corrientes favorables sobre una determinada cuestión, de ahí que tengamos la obligación de expandir la cultura de la vida. Como intuye acertadamente el pensador mexicano Rodrigo Guerra, la auténtica batalla que se va a librar se dará en el plano cultural más que político:

La batalla principal no es de orden político sino cultural. Para que los eventuales triunfos políticos a favor de la vida no sean débiles o efímeros, se requiere de un trabajo en el campo de la educación, de la conciencia, de las actitudes y de los compromisos vitales. La batalla que estamos librando es primariamente cultural antes que política (21).

Es importante reseñar que una persona que haya practicado el aborto debe siempre ser tratada con caridad y se le han de tender puentes de ayuda, pues defendemos lo humano en su particularidad. Otra cosa distinta es admitir la ideología abortista a la que es necesario hacerle frente desde la razón y los argumentos científicos de una forma pacífica. Una antiideología se presenta como aparente ideología -como algunas que se han sucedido en nuestra historia más reciente-, pero con la contrapartida de que se vuelve contra el ser humano y se levanta contra él, especialmente contra la vida de los más débiles que toda sociedad debe proteger si quiere ser tenida como ejemplar. El aborto es antisocial porque destruye la solidaridad genérica a la vez que destruye la filiación. La solidaridad que tiene que mantener una generación con otra se fragmenta con el aborto, o sea, el cuidado que los padres deben a los hijos se malogra y de ahí surgen otras graves manifestaciones de insolidaridad generacional. Es decir, ¿̇por qué una generación se sacrifica tanto para sacar adelante a la siguiente? La respuesta sería interesante y sigue siendo un reto en el nivel de comprensión para la sociología, pero con el aborto se rompe este equilibro protector de los padres y se pasa desgraciadamente a la eliminación del hijo.

\section{LOS TRIBUNALES INTERNACIONALES EN LA DENUNCIA DEL DERECHO AL ABORTO}

El aborto traspasa las fronteras políticas porque es un atentado contra los derechos humanos. Las transgresiones a cualquier derecho, por ser humano, no pueden estar justificadas por las fronteras políticas; más bien, tales 


\section{EN DEFINITIVA, HAY QUE HABLAR MUCHO MÁS DEL ABORTO, ESPECIALMENTE EN FOROS \\ ACADÉMICOS, Y DESEAMOS RESALTAR QUE CON ELLO NO HACEMOS POLÍTICA, SINO QUE \\ DEFENDEMOS EL PRIMERO DE LOS DERECHOS}

HUMANOS: EL DE LA VIDA.

derechos han de estar protegidos por estas. De hecho, las distintas violaciones e injusticias contra cualquiera de los derechos de la persona han justificado distintos embargos y sanciones a países que los han vulnerado sistemáticamente. Por todo ello, necesitamos una sociedad que en el nivel racional enfatice su voz contra toda injusticia y, en este sentido tenemos que concienciar todavía mucho más al mundo académico. Todas las universidades del mundo deberían tener en su ideario y estatutos la defensa de la vida como estandarte, pues el aborto representa, en cifras, una de las mayores causas de muerte en el mundo, la cual supera a las hambrunas y las distintas catástrofes o epidemias. Existe una diferencia sustancial entre unos casos y otros, pues el hambre, las epidemias y los desastres naturales no son queridos, mientras que el aborto es buscado y, en muchos casos, potenciado. Cada año se practican en el mundo 55,7 millones de abortos, según un informe de la Organización Mundial de la Salud (OMS). La mayoría de ellos se producen en Asia, África y América Latina (22). No obstante, deberíamos tener una mirada de esperanza ante todos estos datos y sinergias; hay ejemplos que pueden dar algunas luces para aliarse con la causa de la vida. Así, los Tribunales Internacionales son eficaces en múltiples causas de guerras y crímenes contra la humanidad, como el caso europeo de la guerra en la extinta Yugoslavia, donde han sido juzgados por crímenes de guerra y genocidios distintos dirigentes que tomaron decisiones homicidas contra el pueblo. En Francia, el senado ha declarado como delito penal negar el holocausto armenio (que supuso uno de los mayores genocidios del siglo XX), a sabiendas de que Turquía podía tomar represalias políticas por esta declaración y poner en peligro la estabilidad del marco común europeo. La corte alemana, a su vez, condenó a una mujer a seis meses de cárcel por negar el Holocausto (negar el Holocausto, en el que murieron seis millones de judíos, es un delito ahora en Alemania, y puede suponer hasta cinco años en prisión). Todo esto es delito en estos países desde el punto de vista jurídico, y sin duda en lo concerniente a la denuncia del aborto por parte de los tribuales internacionales podría serlo en el futuro si se despeja una vía por donde la fuerza del valor de la dignidad de toda vida humana se abra camino. El primer paso sería reconocer el grave daño que causa admitir el aborto en el terreno conceptual, y el segundo, denunciar políticas abortivas que se suceden en el mundo. En definitiva, hay que hablar mucho más del aborto, especialmente en foros académicos, y deseamos resaltar que con ello no hacemos política, sino que defendemos el primero de los derechos humanos: el de la vida. Para ello tenemos que incidir en las bases sociales de modo que estas asuman su responsabilidad social y exijan de sus servidores políticos una verdadera justicia:

La salud social depende más de lo civil que de lo político. Más de las familias que de los Estados; más de las comunidades que de los gobiernos; más de las relaciones libres que de las directivas burocráticas. Estamos convencidos que ese trasiego puede darse si intervenimos para que al menos se dé dentro de nosotros (23). 
Desde el mundo académico tenemos que hablar de lo que el aborto supone, no con la idea de ser doctrinarios ni sentar ningún tipo de postura inflexible al respecto, ni mucho menos acusar a nadie en particular, pero sí con la firme intención de arrojar luz sobre un problema social que se acrecienta y ante el cual la sociedad parece acostumbrase sin una suficiente base crítica. Aquí se da una vulneración del derecho a la vida, y esa transgresión hay que señalarla con el ánimo de darle solución. Esta defensa nos beneficia a todos. Como se señala desde el terreno de las ciencias sociales, aquellas sociedades que protegen la vida presentan índices más óptimos de salud social entre los jóvenes y viceversa:

Los sociólogos han estudiado este punto, y específicamente el caso irlandés, para concluir que las sociedades que han adoptado una actitud no permisiva frente al aborto son también las sociedades donde la calidad de vida infantil es superior. Paradójicamente, los países abortistas, y paradigmáticamente los EE.UU., sufren de graves disfunciones en la socialización de los jóvenes (12).

Desde la órbita política, el actual presidente francés, Emmanuel Macron, confesó el pasado 9 de abril que Europa necesita reconstruir su tejido social y la calidad de los valores que son necesarios, por lo que tender puentes entre unos y otros es es hoy ineludible. Tesis a la que también parece sumarse la actual canciller de Alemania Ángela Merkel. Es, por tanto, posible un viento de cambio en nuestra sociedad a fin de que la voz de aquellos que defienden la dignidad de toda vida, desde posturas científicas, empiece a ser escuchada en aquellos atrios y lugares de encuentro que debemos potenciar.

\section{CONCLUSIONES}

1. La dimensión social de lo humano debe ser subrayada en el actual mundo académico. Con ello se enriquece la comprensión antropológica de un sujeto que es social desde que es engendrado. La familia es el lugar desde donde se entiende la diacronía como una parte constitutiva de nuestra realidad. En esta institución se gesta el ser humano y es ahí donde se establecen los rasgos constitutivos que nos identifican como tales: fraternidad, solidaridad intergeneracional, amor. No obstante, la familia presenta en la actualidad graves disfunciones que es necesario corregir en orden a restablecer una adecuada salud social; estas que nos remiten a una serie de criterios morales de excelencia.

2. Lafiliación es un dato social humano que nos identifica a todos sin excepción como miembros del género humano. Tal realidad nos permite un diálogo intergeneracional que nos faculta para desarrollar el afecto y la protección, cualidades específicamente humanas. La presencia de la violencia en el seno familiar es algo extraño a su naturaleza, pues de ella se espera la protección y la seguridad. Por ello el aborto se presenta como una de las graves disfunciones sociales en la actualidad, y como una realidad contradictoria en términos conceptuales.

3. La verdadera defensa de la salud social y de la familia se libra en el terreno de las ideas y esta merece ser librada con rigor y valor. Es necesario huir de los eufemismos en un primer momento y debatir sobre la realidad veladamente para que esta pueda ser digerida. Acostumbrarnos al eufemismo o a la mala praxis conceptual significa abrir la puerta a justificar realidades injustificables y, peor aún, acostumbrarnos a ellas como 
sociedad. Con ello queremos decir que la cuestión del aborto y la permisividad a la hora de eliminar los vínculos de filiación nos conducen a una progresiva deshumanización. Nos estamos acostumbrando a una vida en sociedad cada vez más cosmética y líquida, pero porque hemos permitido que se desvirtúe el concepto que señala la realidad o la disfraza.

4. El abortismo como ideología es profundamente antisocial y se hace necesario hacerle frente en el plano de las ideas. Por tanto, pretendemos generar renovadas sinergias de opinión a favor de la dignidad de la vida humana y abogamos para que cada vez sean más los actores que se sumen a esta defensa. La gran desigualdad social en la actualidad se sitúa entre los que son y los que no son en el plano vital. Por ello, edificar una sociedad sensible a las crecientes injusticias es uno de nuestros mayores logros como intelectuales.

5. Tenemos a nuestro favor la fuerza y belleza de la verdad que se abren camino, aunque la corrección política y los modernos medios de comunicación social se impongan y ejerzan una presión a la que no resulta fácil sustraerse. El avance social está en la búsqueda de la virtud, no exclusivamente del progreso material y el desarrollo de la técnica, por lo que tenemos la misión de ganar el futuro a nuestros hijos y de establecer cauces de dignidad hacia una sociedad mejor. La tarea ha de centrarse en la eliminación de toda violencia y en el intento de neutralizar el hecho de que alguien tenga potestad para decidir sobre la vida de un tercero.

6. Hacemos un llamado a los tribunales internacionales para que se sumen a este noble ideal de la defensa de los más débiles. Somos conscientes de que la sociedad es sensible a las injusticias que crecen día a día, y alzamos la voz para que la realidad del aborto sea tenida como una de las más graves. Del mismo modo, todas y cada una de las universidades deberían tener como el primero de sus objetivos la defensa de la dignidad de la vida humana en todas sus fases. La salud que como sociedad buscamos se basa en estas premisas y la búsqueda de una sociedad más humana se establece sobre la defensa de aquellos que no pueden defenderse por sí mismos.

Conflicto de intereses: ninguno declarado.

$$
\text { REFERENCIAS }
$$

1. Aznar J. La vida humana naciente. Madrid: BAC.

2. Pérez-Adán J. Comunitarismo. Cultura de solidaridad. Madrid: Sekotia; 2003.

3. Marías J. Antropología metafísica. Madrid: Alianza; 1995.

4. Gevaert J. Introducción a la antropología filosófica. Salamanca: Sígueme; 2008.

5. Pérez-Adán J. Repensar la familia. Pamplona: Eiunsa; 2005.

6. Pérez-Adán J. 7 conceptos clave de la sociología actual. Valencia: Fundación Interamericana Ciencia y Vida; 2017.

7. Marías J. La cuestión del aborto. Madrid: ABC; 1994.

8. Pérez-Adán J. Adiós Estado, bienvenida comunidad. Pamplona: Eiunsa; 2008.

9. López-Moratalla N. "La realidad del embrión humano en los primeros quince días de vida”. pers.bioét. 2004;7(20-21):6-23. Disponible en: http://personaybioetica.unisabana.edu.co/index.php/personaybioetica/article/view/882

10. Pérez-Adán J. La salud social. Madrid: Trotta; 1999. 
11. ABC. La soledad, el nuevo factor de riesgo cardiovascular. 2018 [visitado 2018 abr 3]. Disponible en: http://www.abc.es/ salud/enfermedades/abci-soledad-nuevo-factor-riesgo-cardiovascular-201803270144_noticia.html

12. Pérez-Adán J. Manifiesto anticonservador. Granada: Carmaiquel; 1998.

13. Instituto Nacional de Estadística. El número de nacimientos disminuyó un 2,8\% respecto al año 2015 y el de defunciones se redujo un 3,2\%. 2017 [visitado 2018 jun 05]. Disponible en: http://www.ine.es/prensa/mnp_2016_p.pdf

14. Aciprensa. ¿Sabes cuántos millones de abortos se hacen al año en China? 2018 [visitado 2018 abr 25]. Disponible en: https:// www.aciprensa.com/noticias/sabes-cuantos-millones-de-abortos-se-hacen-al-ano-en-china-47919

15. Arana I. La natalidad solo repunta un $11,5 \%$ en China un año después del fin de la política del hijo único. 2017 [visitado 2018 mar 31]. Disponible en: http://www.elmundo.es/socieda d/2017/01/23/58861f29e5fdea05028b45bd.html

16. Observatorio de Bioética. Sigue aumentando el número de abortos en el mundo. 2016 [visitado 2018 jun 22]. Disponible en: http://www.observatoriobioetica.org/2016/05/aumenta-elnumero-de-abortos-mundo/13945
17. Toledo O. El drama de los ancianos abandonados por sus familias. 2018 [visitado 2018 jul 15]. Disponible en: https:// politica.elpais.com/politica/2018/03/26/diario_de_espana/1522092904_996135.html

18. García-Morente M. Ensayos sobre el progreso. Madrid: Encuentro; 2002.

19. Boltanski L. La condición fetal. Una sociología del engendramiento y del aborto. Madrid: Akal; 2016.

20. Ballesteros J. Que sea integral la ley contra la violencia de género. 2010 [visitado 2018 may 03]. Disponible en: http://eresdelosquepiensan.org/tag/BALLESTEROS/page/2/

21. Guerra R. El principal reto que plantea el aborto en México es de orden cultural. 2008 [visitado 2018 feb 23]. Disponible en: http://es.catholic.net/op/articulos/4231/cat/261/el-principal-retoque-plantea-el-aborto-en-mexico-es-de-orden-cultural.html\#

22. Lucio C. 25 millones de abortos inseguros al año en el mundo. 2017 [visitado 2018 jun 17]. Disponible en: http://www. elmundo.es/ciencia-y-salud/salud/2017/09/28/59cb8f8222601 d05598b4675.html

23. Pérez-Adán J. Caridad política. Valencia: Fundación Interamericana Ciencia y Vida; 2017. 